\title{
Training in Safe Opioid Prescribing and Treatment of Opioid Use Disorder in Internal Medicine Residencies: a National Survey of Program Directors
}

\author{
Donna M. Windish, MD, MPH' (D), Jillian S. Catalanotti, MD, $M P H^{2}$, Aimee Zaas, $M D, M H S^{3}$, \\ Michael Kisielewski, MA ${ }^{4}$, and John P. Moriarty, $M D^{7}$ \\ 'Yale Primary Care Residency Program, Yale New Haven Health, Yale University School of Medicine, St. Raphael's Campus, 1450 Chapel Street, \\ Private 304, New Haven, CT, USA; ${ }^{2}$ The George Washington University School of Medicine and Health Sciences, Washington, DC, USA; ${ }^{3}$ Duke \\ University School of Medicine, Durham, NC, USA; ${ }^{4}$ Alliance for Academic Internal Medicine, Alexandria, VA, USA.
}

\begin{abstract}
BACKGROUND: Training future clinicians in safe opioid prescribing (SOP) and treatment of opioid use disorder (OUD) is critical to address the opioid epidemic. The Accreditation Council on Graduate Medical Education requires all programs to provide instruction and experience in pain management and will mandate addiction medicine clinical experiences for internal medicine trainees.
\end{abstract}

OBJECTIVE: Assess residents' training in SOP and treatment of OUD and identify training barriers.

DESIGN: Cross-sectional nationally representative survey was emailed in 2019.

PARTICIPANTS: Four hundred twenty-two Association of Program Directors in Internal Medicine members in US internal medicine residency programs.

MAIN MEASURES: Program opportunities and challenges to developing or implementing training in SOP, treatment of OUD, and buprenorphine waiver training, and perceived curricular effectiveness.

KEY RESULTS: The response rate was 69.4\% (293/422). Most programs required didactics in SOP (94.2\%) and treatment of OUD (71.7\%). Few programs required clinical experiences including addiction medicine clinics (28/ $240,11.7 \%$ ), inpatient consult services (11/240, 4.6\%), or offsite treatment rotations $(8 / 240,3.3 \%)$. Lack of trained faculty limited developing or implementing curricula $(61.5 \%)$. Few respondents reported that their program was "very effective" in teaching SOP $(80 / 285,28.1 \%)$ or treatment of OUD $(43 / 282,15.3 \%)$. Some programs offered buprenorphine waiver training to residents (83/ $286,29.0 \%)$ and faculty $(94 / 286,32.9 \%)$ with few mandating training (11.7\% (28/240) and 5.4\% (13/240) respectively). Only 60 of 19,466 (0.3\%) residents completed buprenorphine waiver training. Primary care programs/ tracks were more likely to offer waiver training to residents (odds ratio [OR], 3.07; 95\% CI, 1.68-5.60; $P<0.001$ ) and faculty (OR, 1.08; 95\% CI, 1.01-3.22; $P=0.05$ ).

CONCLUSIONS: In this nationally representative survey, few internal medicine residency programs provided clinical training in SOP and treatment of OUD, and training was not viewed as very effective. Lack of effective training

Prior Presentations None.

Received April 10, 2021

Accepted August 13, 2021

Published online November 2, 2021 may have adverse implications for patients, clinicians, and society.

KEY WORDS: graduate medical education; safe opioid prescribing; treatment of opioid use disorder.

J Gen Intern Med 37(11):2650-60 DOI: $10.1007 / \mathrm{s} 11606-021-07102-y$

(C) Society of General Internal Medicine 2021

\section{INTRODUCTION}

An estimated 10.1 million Americans used opioids either medically or non-medically in $2019 .{ }^{1}$ During the 12 months ending in May 2020, over 80,000 deaths occurred in the USA due to opioid overdose. ${ }^{2}$ Increases in opioid use led the US Department of Health and Human Services to declare the opioid crisis to be a public health emergency. ${ }^{3}$ Opioid use and overdose deaths have sharply increased since the COVID19 pandemic, ${ }^{2,}{ }^{4}$ which created new social isolation, unemployment, ${ }^{5}$ and a strained healthcare system.

Internists are on the front lines of treating opioid use disorder (OUD) as they have established relationships with patients and are trained in chronic disease management. Although many providers have favorable perceptions of treating OUD, ${ }^{6}$ most general internists do not feel prepared to screen, diagnose, or treat patients with substance use disorders. ${ }^{7}$ Educational strategies are required to ensure clinicians are trained in safe opioid prescribing (SOP) for acute and chronic pain and the evaluation and treatment of patients with OUD. In 2019, the Accreditation Committee for Graduate Medical Education (ACGME) required all programs "to provide instruction and experience in pain management" and recognize signs of addiction. ${ }^{8}$ Subsequently, the 2022 ACGME program requirements will require didactic and clinical experiences in addiction medicine for all internal medicine trainees. ${ }^{9}$

Internal medicine residents commonly care for patients with OUD and chronic pain, many of whom are on long-term opioid therapy. ${ }^{10}$ Some training programs have successfully taught residents how to address chronic pain and SOP. ${ }^{11-13}$ Unfortunately, a minority of internal medicine, psychiatry, and family medicine programs offered buprenorphine training in 
2013. ${ }^{14}$ Not surprisingly, one program's internal medicine residents felt unprepared to treat addiction in 2013, despite caring for many patients with substance use disorder. ${ }^{15}$ Given the rapid rise of the opioid epidemic, these studies may underestimate training challenges today.

To understand current training in residency programs related to SOP and treatment of OUD, we incorporated questions about these topics into a national survey of internal medicine residency program directors. Additionally, we aimed to identify potential barriers to education in SOP and treatment of OUD.

\section{METHODS}

\section{Study Design, Setting, and Participants}

The Association of Program Directors in Internal Medicine (APDIM) oversees an annual survey of internal medicine residency program directors studying multiple themes central to graduate medical education (GME) training. The 2019 survey was disseminated to program directors from all 422 APDIM member programs with ACGME accreditation prior to July 1, 2018, representing $82 \%$ of US ACGME-accredited internal medicine programs.

\section{Instrument}

The annual APDIM survey methods have been previously described. ${ }^{16}$ Questions specific to SOP and treatment of OUD were pretested, pilot-tested, and revised by the APDIM Annual Survey and Scholarship Committee, six experts in GME and two experts in addiction medicine. Questions were finalized after an iterative process of these groups until no other changes in questions were deemed necessary (Appendix 1 in the Supplementary Information). Questions asked about training experiences offered and required in SOP and treatment of OUD, curricular effectiveness (5-point scale from "very ineffective" to "very effective"), challenges to developing or implementing training, whether and how programs offer and support medication-assisted treatment (MAT)/ buprenorphine waiver training for residents and faculty, number of residents who have completed MAT/buprenorphine waiver training, and opinions of whether training in SOP and treatment of OUD should be required (5-point scale from "strongly disagree" to "strongly agree").

\section{Statistical Analysis}

We obtained additional training program data from the US Census Bureau, ${ }^{17}$ ACGME,${ }^{18}$ and American Medical Association ${ }^{19}$ for different program characteristics (see Table 1 for

Table 1 Core Characteristics of Responding and Nonresponding Internal Medicine Residency Programs from the 2019 Survey of US Internal Medicine Residency Program Directors

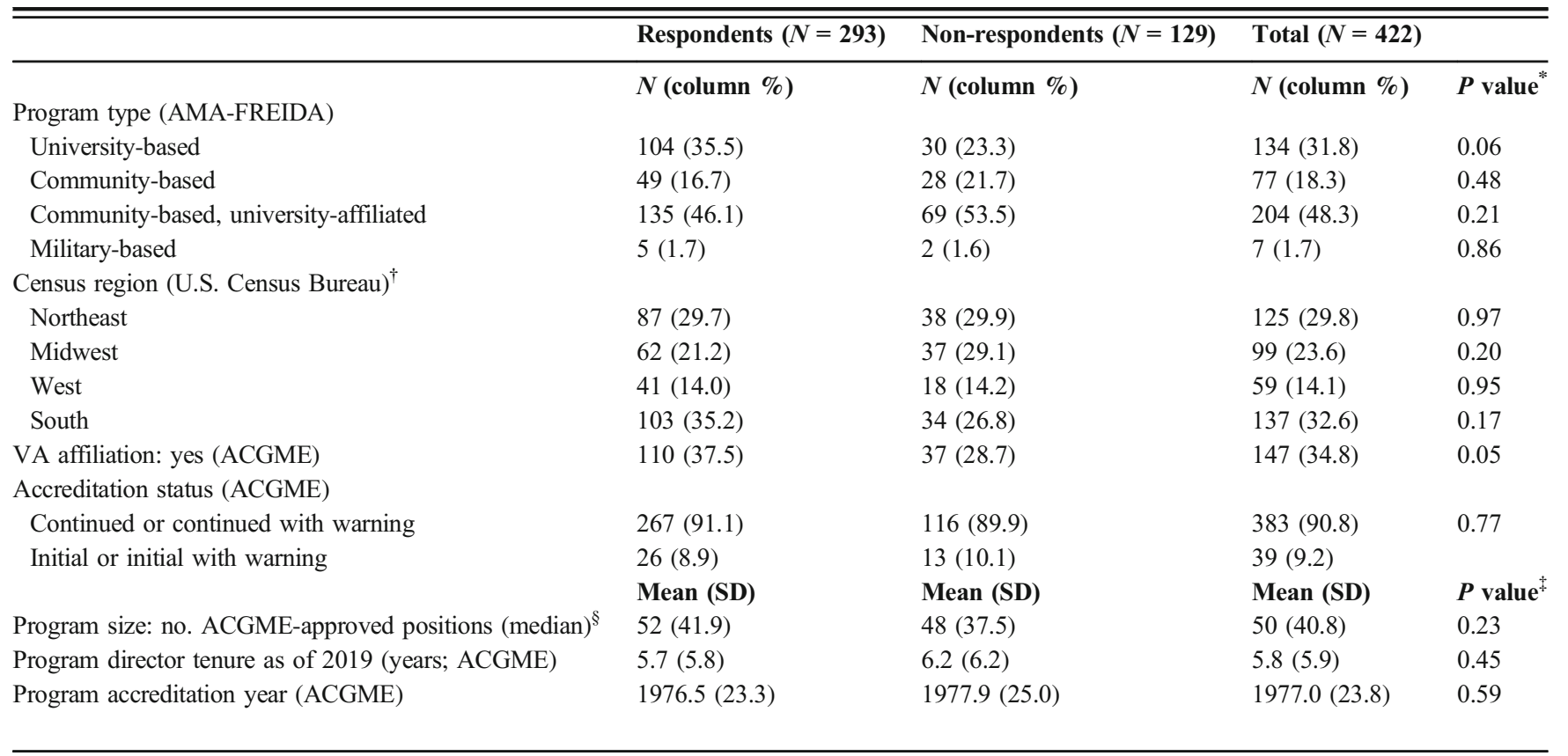

AMA-FREIDA, American Medical Association Residency and Fellowship Database; ACGME, Accreditation Council for Graduate Medical Education; ABIM, American Board of Internal Medicine; VA, Veterans Affairs; SD, standard deviation

*Bivariate (adjusted Wald [Pearson]) test of association with one degree of freedom used for categorical variables

${ }^{+}$Excludes programs from two US territories, due to small cell sizes/data confidentiality

${ }^{\ddagger}$ Welch's t-test

${ }^{\S}$ Equality-of-medians test (continuity corrected Pearson chi-square) 


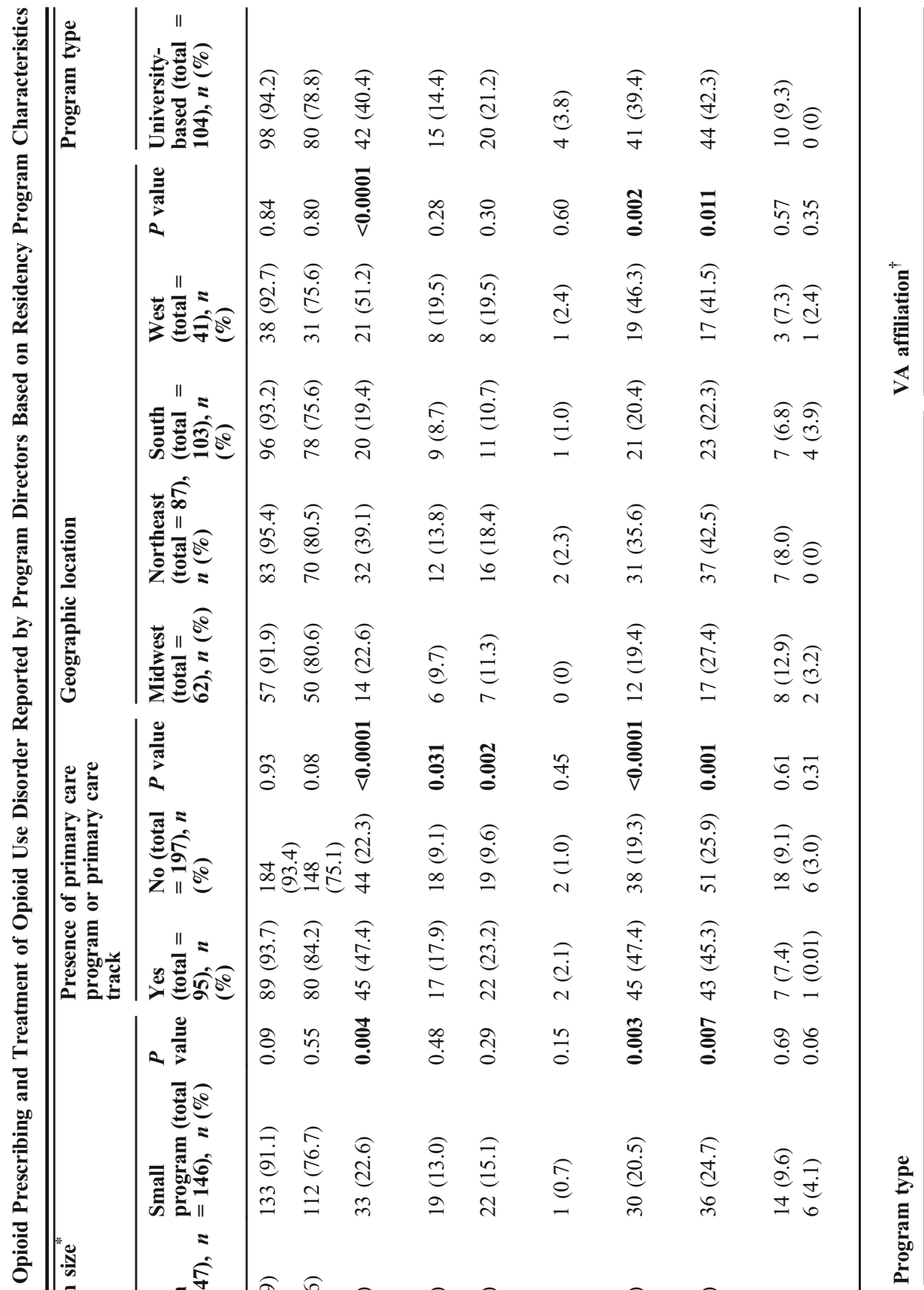

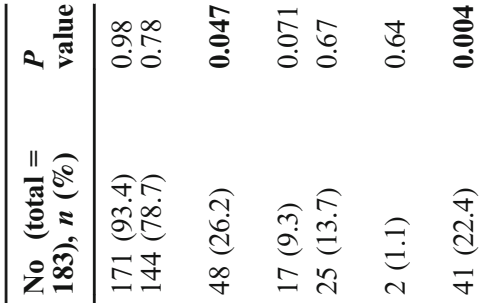

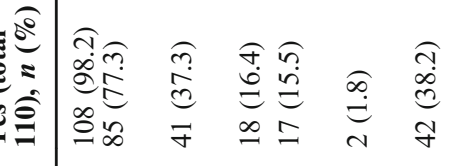

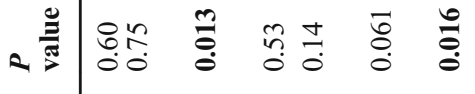

플

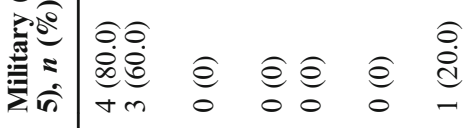

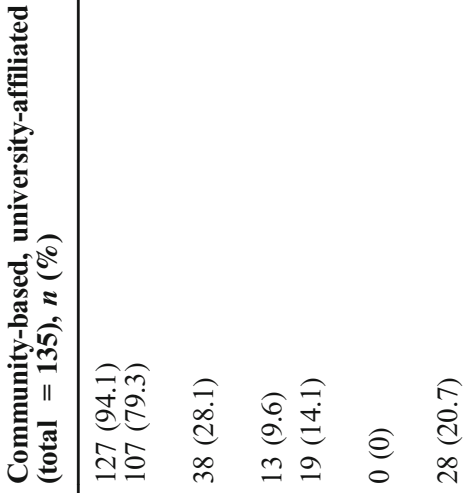

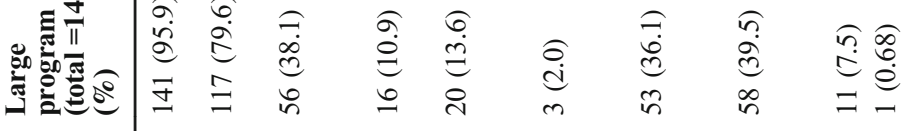

I

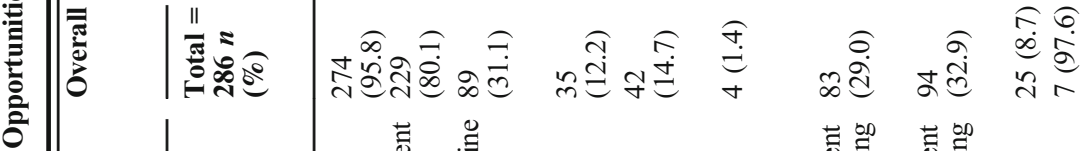


specific characteristics used from each source). Data were analyzed using Stata 16 SE (StataCorp, College Station, TX).

We compared essential characteristics of respondents and their programs to the complete survey population to assess response representativeness using adjusted Wald (Pearson) test of association and Welch's $t$-test (due to unequal variances). Subgroup analyses were conducted based on factors believed to influence training experiences as recommended by experts in GME and addiction medicine, including residency program type (university-based, community-based, community-based university-affiliated, and military), geographic region, Veterans Administration (VA) affiliation, primary care program/track, and program size $(\geq$ or $<52$ residents, the median number for responding programs). Bivariate analyses were conducted using Fisher's exact or chi-square tests. Logistic regression reporting odds ratios were used to determine the influence of the above factors on training offerings and barriers to training. All statistical tests were 2-sided, and $P<0.05$ was considered statistically significant.

The study was deemed exempt by Pearl IRB (US DHHS \#IRB00007772). The survey was conducted from August 12 through December 9, 2019, and included five email reminders to non-respondents.

\section{RESULTS}

The survey response rate was $69.4 \%$ (293/422). There was no statistical association between respondents and nonrespondents based on any program characteristic (Table 1).

\section{Offered Training in SOP and Treatment of OUD}

Although most programs (286/293, 97.6\%) reported offering some training in SOP and treatment of OUD, programs differed in the type and number of training opportunities. Of the 286 programs offering some training, most offered didactic curricula in SOP $(n=274,95.8 \%)$ and treatment of OUD (229, $80.1 \%$ ) (Table 2). Fewer offered clinical training in addiction medicine $(113,39.6 \%)$, with $31.1 \%$ of programs offering rotations in a dedicated addiction medicine clinic. Few programs offered training on inpatient addiction consult services $(42,14.7 \%)$ or in offsite opioid treatment facilities $(35,12.2 \%)$ (Figure 1). Larger programs ( $\geq 52$ residents), university-based programs, and those in the West offered more opportunities in addiction medicine clinics within the program/hospital $(38.1 \%, 40.4 \%$, and $51.2 \%$ respectively) (Table 2). Primary care programs/tracks more commonly offered training in addiction medicine clinics within the program/hospital (45/95, $47.4 \%$ compared to $44 / 19722.3 \%, P<0.001$ ), in offsite opioid treatment facilities $(17 / 95,17.9 \%$ compared to $18 / 197,9.1 \%$, $P=0.03$ ), and on addiction medicine inpatient consult services (22/95, 23.2\% compared to $19 / 197,9.6 \%, P=0.002)$. A higher percentage of residency programs with VA affiliations 


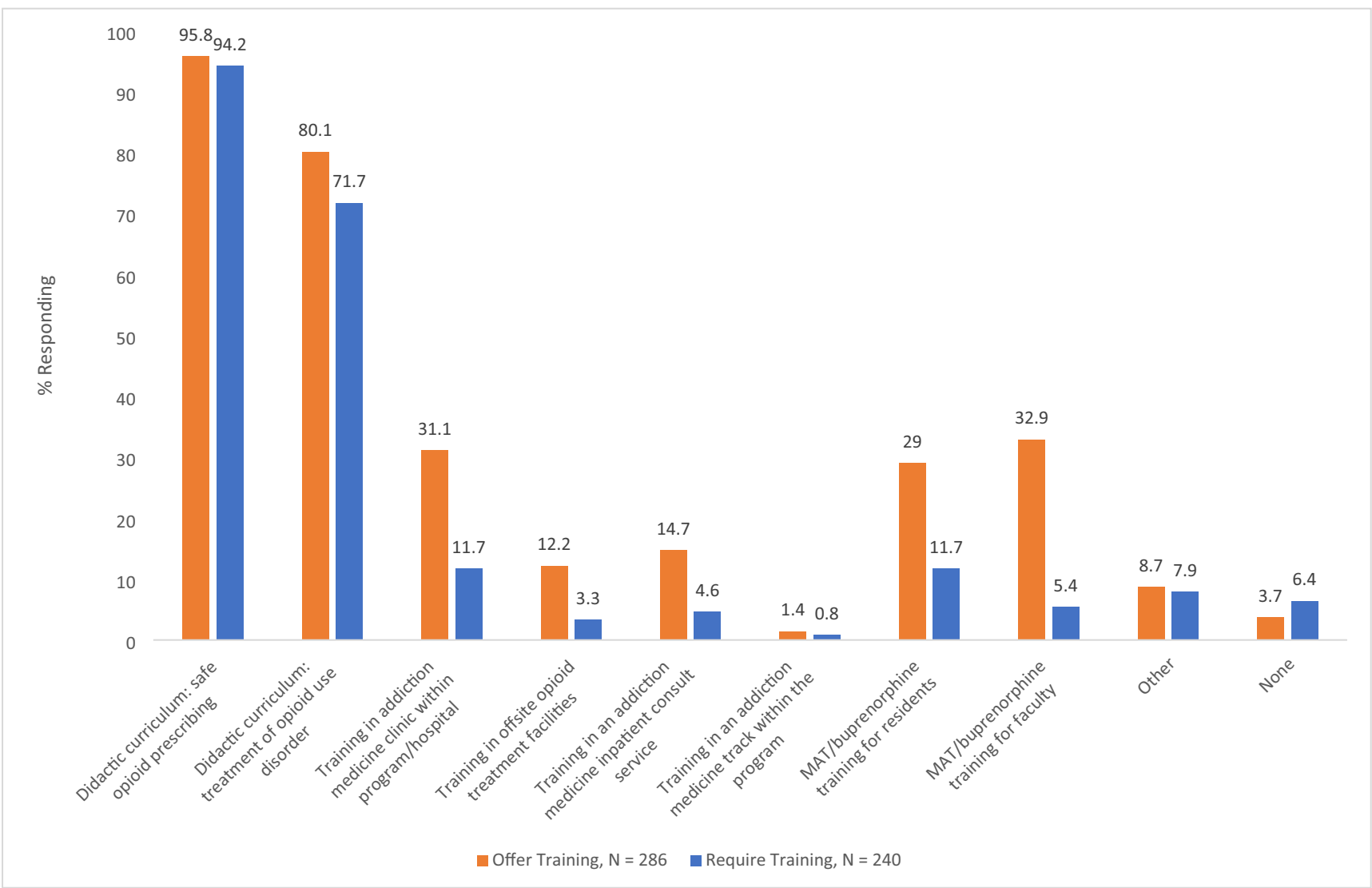

Fig. 1 Program offerings and required training in safe opioid prescribing and treatment of opioid use disorder in 286 responding internal medicine residency training programs

offered training in addiction medicine clinics within the program/hospital compared to non-VA affiliated programs

(41/110, 37.3\% compared to $48 / 183,26.2 \%, P=0.05)$.

In multivariate analyses, programs in the Northeast and West were more likely to offer training in addiction medicine clinics compared to programs in the South (odds ratio [OR], 2.53; 95\% CI, 1.22-5.23; $P=0.01$ and OR, 4.98; 95\% CI, $2.15-11.56$, respectively; $P<0.001$ ), and primary care programs/tracks were more likely than non-primary care programs/tracks to offer training in addiction medicine clinics (OR, 2.45; 95\% CI, 1.36-4.41; $P=0.003$ ) and addiction medicine inpatient consult services (OR, 2.47; 95\% CI, $1.17-5.20 ; P=0.02)$. No other differences were determined in any subgroup or multivariate analyses.

\section{Required Training in SOP and Treatment of OUD}

Most programs $(240 / 293,81.9 \%)$ required some training in SOP and treatment of OUD. Of the 240 programs, many required didactic training in SOP $(94.2 \%)$ and treatment of OUD (71.7\%) (Figure 1). Beyond didactics, few programs required dedicated clinical training in SOP and treatment of OUD including in addiction medicine clinic rotations within the program/hospital (11.7\%), inpatient addiction consult rotations $(4.6 \%)$, or training in offsite opioid treatment facilities $(3.3 \%)$. A higher percentage of programs in the Northeast and West required training in an addiction medicine clinic (14.9\% and. $14.6 \%$ compared to $9.7 \%$ in the Midwest and $2.8 \%$ in the South, $P=0.02$ ). More programs in the West required residents to train offsite in opioid treatment facilities $(4 / 41,9.8 \%)$ compared to other regions $(3 / 87,3.4 \%$ Northeast, $1 / 103,1.0 \%$ South, $0 / 62,0.0 \%$ Midwest, $P=0.01)$. No other requirements differed in other subgroup or multivariate analyses.

\section{MAT/Buprenorphine Waiver Training for Residents}

Program directors reported that only 60 of $19,466(0.3 \%)$ internal medicine residents from all programs represented in this survey completed MAT/buprenorphine waiver training. Approximately $30 \%$ of programs offered residents MAT/ buprenorphine waiver training. Of the 108 programs that offered training, $33.3 \%$ provided protected time to complete the entire waiver training and $41.7 \%$ provided partially protected time. Many programs (41.7\%) invited faculty outside of their program/institution to teach. Few programs $(22.2 \%)$ had grant funding to support training, and fewer $(47 / 283,16.4 \%)$ directed residents to obtain training on their 


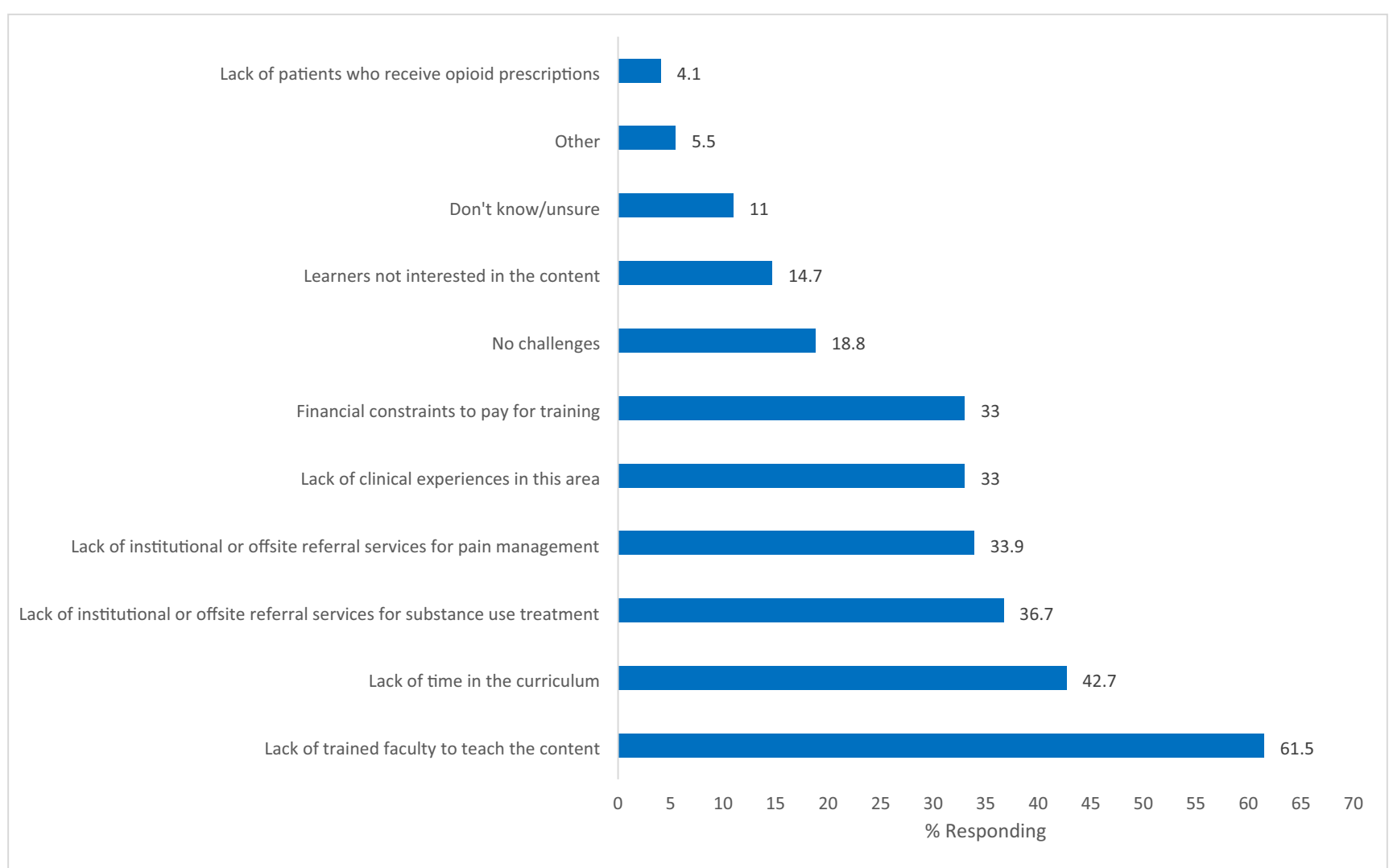

Fig. 2 Challenges to the development or implementation of curricula in safe opioid prescribing and treatment of opioid use disorder in the 218 internal medicine responding residency training programs who noted challenges.

own. Over $27 \%(77 / 283)$ of programs did not offer any instruction, time, direction, or funding for MAT/buprenorphine waiver training. Almost 20\% (51/283) of programs did not allow residents to obtain MAT/buprenorphine training due to lack of faculty to supervise residents in buprenorphine management.

In subgroup analyses, larger training programs more commonly offered MAT/buprenorphine waiver training to residents compared to smaller programs $(36.1 \%$ (53/147) compared to $20.5 \% 30 / 146, P=0.003$ ) (Table 2). University-based programs offered residents MAT/buprenorphine waiver training more than other program types $(39.4 \%$ compared to $\leq$ $26.5 \%$ for all others, $P=0.02$ ). Opportunities for MAT/ buprenorphine wavier training were more common in the Northeast and West $(35.6 \%$ (31/87) and 46.3\% (19/41) respectively compared to $\sim 19.5 \%$ each for the other two regions, $P=0.002)$. Primary care programs/tracks more commonly offered MAT/buprenorphine waiver training than nonprimary care programs/tracks $(47.4 \%$ (45/95) compared to $19.3 \%(38 / 197), P<0.001)$. A higher percentage of programs with VA affiliations offered MAT/buprenorphine training compared to programs without VA affiliations (38.2\% (42/ 110) compared to $22.4 \%(41 / 183), P=0.004)$.

In multivariate analyses, primary care programs/tracks were more likely to offer residents MAT/buprenorphine waiver training than those without (OR, 3.07; 95\% CI, 1.68-5.60; $P$ $<0.001)$. Northeast and West programs offered MAT/ buprenorphine waiver training more than programs in the South (OR, 2.53; 95\% CI, 1.19-5.34; $P=0.02$ and OR, 3.71; 95\% CI, $1.62-8.52 ; P=0.002$, respectively). No other differences were observed in other subgroups or multivariate analyses.

Few programs required MAT/buprenorphine waiver training of their residents $(28 / 293,9.6 \%)$. No statistical differences were seen in required training based on any subgroup or multivariate analyses.

\section{MAT/Buprenorphine Waiver Training for Faculty}

Faculty were offered MAT/buprenorphine waiver training opportunities in $32.1 \%(94 / 293)$ of training programs responding to the survey. Larger training programs offered more opportunities compared to smaller programs $(39.5 \%$ vs. $24.7 \%, P=0.007$ ) (Table 2 ). Primary care programs/ tracks more often offered MAT/buprenorphine waiver training than non-primary care programs/tracks $(45.3 \%$ vs. $25.9 \%, P=0.001)$. A higher percentage of programs in the Northeast and West offered faculty MAT/ buprenorphine waiver training compared to other regions (42.5\% Northeast, $42 \%$ West, $22.3 \%$ South, and $27.4 \%$ Midwest, $P=0.01$ ). A higher percentage of university- 


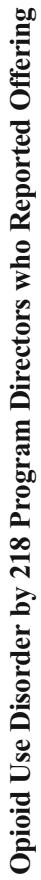

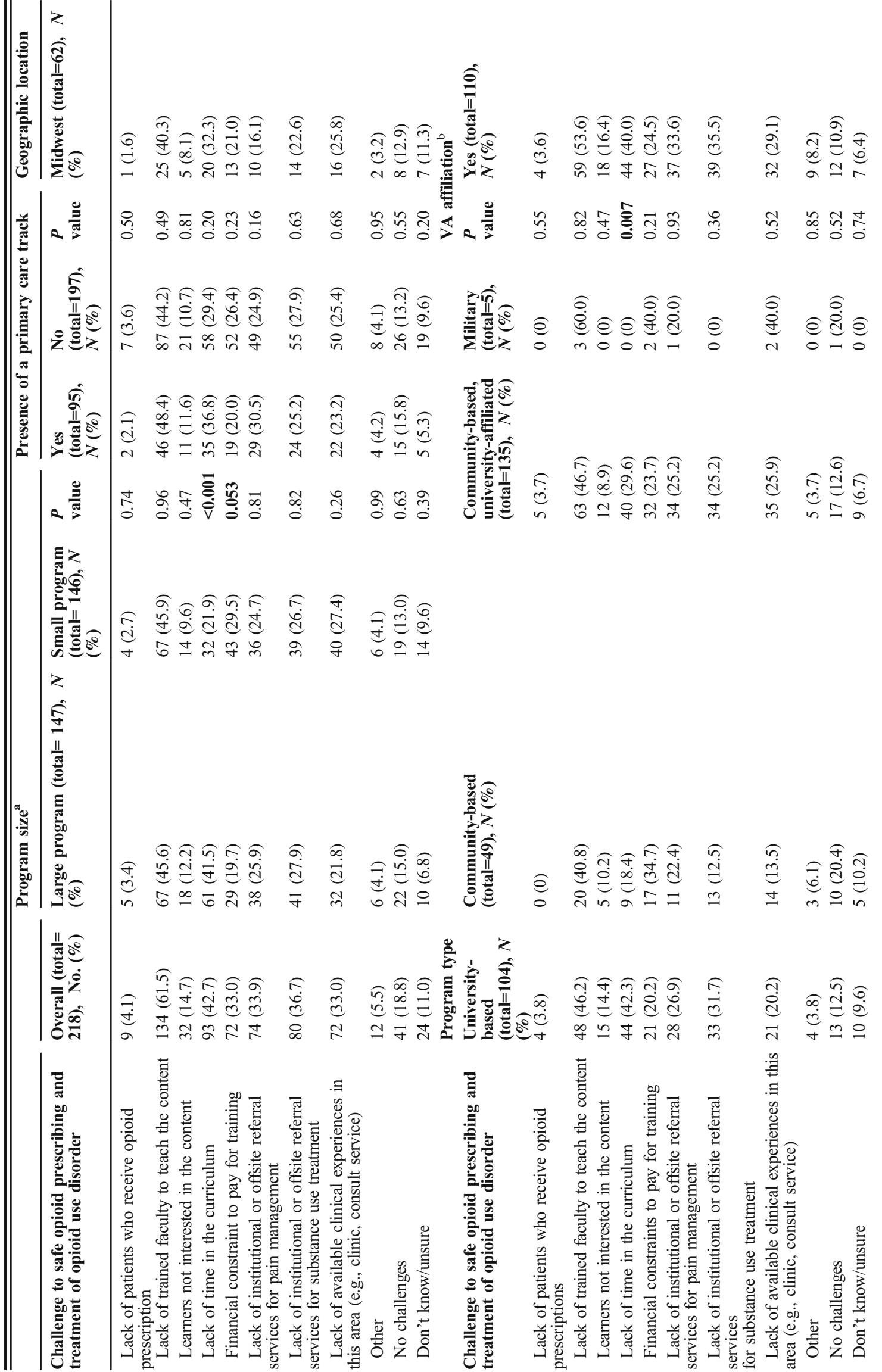




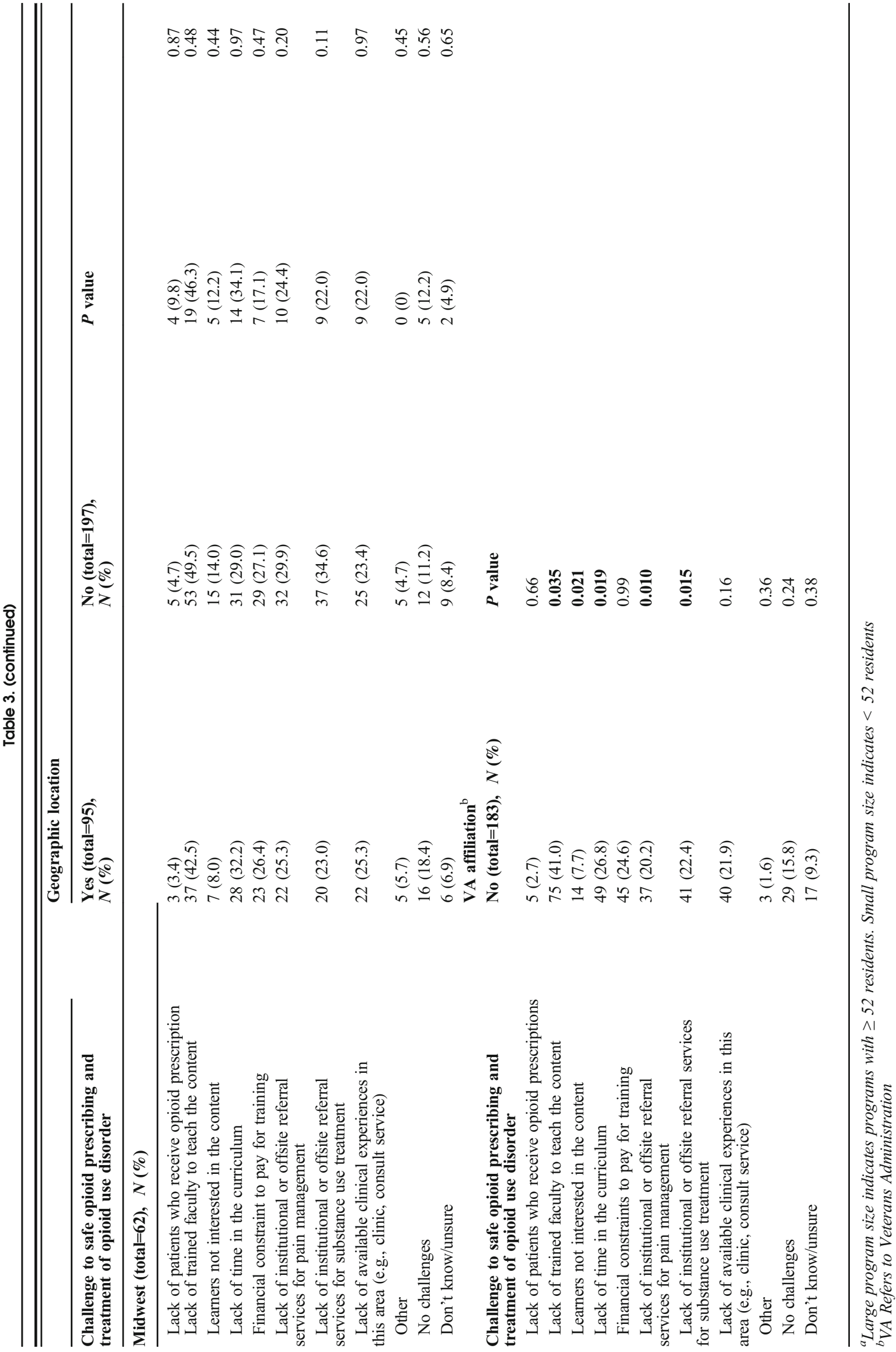


based and military programs offered faculty MAT/ buprenorphine training compared to community-based and community-based university-affiliated programs $(42.3 \%, 40.0 \%, 32.7 \%$, and $23.7 \%$ respectively, $P=$ $0.02)$. A higher percentage of programs with VA affiliations offered faculty MAT/buprenorphine waiver training compared to non-VA affiliated programs (42.7\% compared to $25.7 \%, P=0.002$ ).

In multivariate analyses, primary care programs/tracks were more likely than those without to offer faculty MAT/ buprenorphine waiver training (OR, 1.08; 95\% CI, 1.013.22; $P=0.05$ ). Community-based university-affiliated and military programs more often offered faculty waiver training compared to community-based programs (OR, 3.66; 95\% CI, 1.77-7.59; $P<0.001$ and OR, 2.63; 95\% CI, 1.17-5.92; $P=$ 0.02 , respectively). Programs with VA affiliations also offered more faculty training (OR, 2.27; 95\% CI, 1.17-4.39; $P=$ 0.02 ). No other differences were observed in any other subgroup or multivariate analyses.

Very few programs required MAT/buprenorphine waiver training of their faculty $(5.4 \%)$ (Figure 2 ). No differences were observed in required training in any subgroup or multivariate analyses.

\section{Challenges to Development or Implementation of Curricula in SOP and Treatment of OUD}

Most programs $(218 / 286,76.2 \%)$ reported challenges in curriculum development or implementation of SOP or treatment of OUD. The most common challenge was the lack of trained faculty to teach $(61.5 \%)$ (Figure 2). Other common challenges included lack of curricular time (42.7\%), lack of institutional or offsite referral service for substance use treatment $(36.7 \%)$ or pain management (33.9\%), financial constraints $(33.0 \%)$, and lack of available clinical experiences (33.0\%).

Differences in opinions regarding challenges were observed based on program type and VA affiliation (Table 3). Program directors in university-based programs more often cited lack of curricular time compared to other program types $(42.3 \%$ vs. $29.6 \%$ in community-based university-affiliated and $18.4 \%$ in community-based programs, $P=0.007)$. Program directors with VA affiliations cited more curricular challenges including lack of trained faculty to teach $(53.6 \%$ vs. $41.0 \%, P=0.04)$, curricular time $(40.0 \%$ vs. $26.8 \%, P=0.02)$, institutional or offsite referral services for pain management $(33.6 \%$ vs. $20.2 \%, P=0.01)$, and institutional or offsite referral services for substance use treatment (35.5\% vs. $22.4 \%, P=0.02)$.

\section{Program Effectiveness in Teaching SOP and Treatment of OUD}

A minority of program directors $(80 / 285,28.1 \%)$ reported to believe that their program provided "very effective" education in SOP, with fewer than half (43.8\%) believing their training program provided "somewhat effective" training. In subgroup analyses, a higher percentage of program directors in community-based $(47.8 \%)$ and military programs $(75.0 \%)$ believed their programs were "very effective" compared to program directors in other programs $(26.0 \%$ for communitybased university-affiliated and $20.2 \%$ for university-based, $P$ $=0.001)$. No other differences were observed in other subgroup analyses.

Fewer program directors believed their programs provided effective education in treatment of OUD, with 43.2\% reporting it "somewhat effective" and $15.3 \%$ "very effective." No other differences were seen in any subgroup analysis.

\section{Opinions on Requiring Training in SOP and Treatment of OUD}

Most program directors $(214 / 293,73 \%)$ strongly agreed that a curriculum in SOP should be required for all residents in internal medicine. Only 21 (7.2\%) felt neutral or disagreed that this should be mandated. Fewer respondents (58.4\%) strongly agreed that there should be mandated education in the treatment of OUD. Few program directors $(15.1 \%)$ were neutral or disagreed with requiring this training. No other differences were seen in opinions in any subgroup analysis.

\section{DISCUSSION}

This national survey of internal medicine residency program directors revealed that few internal medicine training programs provided residents with hands-on clinical training in SOP and treatment of OUD and few program directors believed that their programs were very effective in providing this education. Many program directors noted educational barriers, including lack of trained faculty, which was the largest educational barrier reported. Not all program directors believed that residency training should include mandated education in SOP and treatment of OUD. These challenges pose significant hurdles to compliance with the current ACGME requirements, upcoming 2022 ACGME requirements for clinical experiences in addiction medicine, and creation of a workforce competent to provide SOP and treatment of OUD.

Internal medicine residents see a disproportionate number of patients with chronic pain, many on long-term opioid therapy. ${ }^{10}$ Lack of training in how to manage chronic pain, including education in $\mathrm{SOP}^{20}$ may result in decreased confidence in treating chronic pain ${ }^{21}$ and unfavorable opinions of primary care as a career. ${ }^{22}$ Nevertheless, dedicated training in SOP can improve residents' confidence and self-reported practices. ${ }^{12,23,24}$ As noted in our survey, a lack of training of SOP by faculty was the leading educational barrier in resident training. Numerous studies demonstrate barriers and negative attitudes by physicians in prescribing buprenorphine, ${ }^{25-27}$ including limited education, 
unfavorable attitudes about prescribing with fears of diversion, and unfavorable perceptions of patients seeking treatment. ${ }^{25,} 26$ Many barriers could be overcome with adequate MAT/ buprenorphine training ${ }^{27}$ and mentorship programs. ${ }^{28}$ Our findings indicate that training in SOP and OUD is needed for both residents and faculty.

A higher percentage of primary care programs/tracks provided hands-on training in SOP and treatment of OUD. This is encouraging, as residents from these programs are more likely to enter primary care practice and as such, encounter patients with chronic pain and OUD. ${ }^{29}$ Family medicine residents represent another source of future primary care providers. A similar survey of family medicine residency program directors from 2015 mirrored our findings such that their program directors reported a similar lack of trained faculty as the largest educational barrier to having a curriculum in addiction medicine, and a small percentage of family medicine programs $(28.6 \%)$ required an addiction medicine curriculum, ${ }^{30}$ further highlighting the need for improved residency training.

Community-based programs less commonly provided training in SOP and treatment of OUD compared to universitybased, community-based university-affiliated, and militarybased programs, possibly because university-based programs may have more resources to provide ambulatory teaching. ${ }^{31}$ Nevertheless, no training program operates independently. Sponsoring institutions and organizations contribute to resident training opportunities and may have constraints contributing to lack of training experiences.

Training in SOP and treatment of OUD in programs with VA affiliations is encouraging. Veterans are more likely to suffer from OUD with overdose deaths rates double than that of the general population. ${ }^{32}$ Significant efforts at the VA recently aimed to address these increased needs of veterans. In 2017, a jointly supported National Institutes of Health, Department of Defense, and VA initiative commenced addressing cost-effective clinical research for nonpharmacologic approaches of pain management. ${ }^{33}$ Subsequently, the VA's 15th State of the Art conference addressed effective management of pain and addiction. ${ }^{34}$ Residents who train in the VA system may be positively influenced by these efforts as program directors in the VA reported a higher percentage of residents and faculty offered MAT/buprenorphine training than those not having a VA affiliation.

Only $0.3 \%$ of residents completed MAT/buprenorphine training according to the program directors in our study. Even if more residents obtain training, they cannot attain MAT/ buprenorphine prescribing waivers without obtaining personal, rather than institutional, Drug Enforcement Agency licenses, an uncommon practice among internal medicine residents. With few programs offering faculty MAT/buprenorphine training, this prescribing gap remains unfilled for residents' patients. Training in SOP and treatment of OUD can be obtained without having a waiver. Educational strategies for both residents and faculty have been published and can be used by individuals or programs that need educational curricula. ${ }^{35-38}$

A recent study found that $40 \%$ of US counties do not have a single prescriber available to treat OUD. ${ }^{39}$ To address prescribing deficits, the US Department of Health and Human Services announced in April 2021 that it would expand access to MAT by exempting physicians from certain certification requirements to prescribe buprenorphine. ${ }^{40}$ Under this plan, physicians may be exempt from the certification requirements related to the training needed to treat OUD with buprenorphine and will be able to treat up to 30 patients at one time. Using this exemption could increase the number of faculty prescribing buprenorphine and subsequently supervising and training residents in SOP, thus making it possible for programs to meet the new ACGME requirements for didactic and clinical experiences in addiction medicine.

The strengths of this study include its statistically representative response rate and high item response rate, but it is not without limitations. First, APDIM member programs represented $82 \%$ of all study-eligible, ACGME-accredited programs at the time. Thus, the survey responses are not generalizable to the complete population of ACGME-accredited programs. Second, as a cross-sectional survey, the results cannot be extrapolated to represent curricula changes or opinions since the survey was fielded.

Our nationally representative survey demonstrated that few internal medicine residency programs provide residents with hands-on clinical experiences in SOP and treatment of OUD. This leaves programs underprepared to combat the opioid epidemic and meet the current and upcoming ACGME requirements. Many educational barriers limited training opportunities, with a lack of trained faculty predominating. Lack of resident and faculty training may have adverse implications for patients, clinicians, and accrediting organizations and society. Several findings in our study warrant further investigation, including training differences by region and those seen in primary care programs/tracks. Additional training efforts are required to expand resident and faculty education in SOP and treatment of OUD.

Supplementary Information The online version contains supplementary material available at https://doi.org/10.1007/s11606-02107102-y.

Acknowledgements: The authors wish to thank the staff of the Alliance for Academic Internal Medicine (AAIM) for their technical and administrative support, specifically Jordan M. Ortiz, B.S., AAIM Surveys and Data Senior Specialist.(AAIM) for their technical and administrative support, specifically Jordan M. Ortiz, B.S., AAIM Surveys and Data Senior Specialist.

Corresponding Author: Donna M. Windish, MD, MPH; Yale Primary Care Residency Program, Yale New Haven Health, Yale University School of Medicine, St. Raphael's Campus, 1450 Chapel Street, Private 304, New Haven, CT 06511, USA (e-mail: donna. windish@yale.edu). 


\section{Declarations:}

Conflict of Interest: The authors declare that they do not have a conflict of interest.

\section{REFERENCES}

1. U.S. Department of Health and Human Services. Available from https:// www.hhs.gov/opioids/about-the-epidemic/opioid-crisis-statistics/index. html. Accessed February 22, 2021.

2. Centers for Disease Control and Prevention. Overdose deaths accelerating during COVID-19. Available from https://www.cdc.gov/media/releases/ 2020/p1218-overdose-deaths-covid-19.html. Accessed March 7, 2021.

3. U.S. Department of Health and Human Services. Available from https:// www.hhs.gov/opioids/about-the-epidemic/hhs-response/index.html. Accessed February 22, 2021.

4. Millennium Health's Signals Report ${ }^{\mathrm{TM}}$ COVID-19 Special Edition. Available from https://www.millenniumhealth.com/news/ signalsreportcovid/. Accessed February 22, 2021.

5. News release: the employment situation - January 2021. Washington, DC: Bureau of Labor Statistics, February 5, 2021. Available from https://www. bls.gov/news.release/pdf/empsit.pdf. Accessed on February 22, 2021.

6. McGinty EE, Stone EM, Kennedy-Hendricks A, et al. Medication for opioid use disorder: a national survey of primary care physicians. Ann Intern Med. 2020;173(2): 160-162

7. Wakeman SE, Pham-Kanter G, Donelan K. Attitudes, practices, and preparedness to care for patients with substance use disorder: Results from a survey of general internists. Subst Abus. 2016;37(4):635-641.

8. ACGME Common Core Requirements. Opioid Use Disorder. Available from https://www.acgme.org/What-We-Do/Initiatives/Opioid-Use-Disorder. Accessed on March 15, 2021.

9. ACGME Program Requirements for Graduate Medical Education in Internal Medicine; ACGME-approved major revision: February 7, 2021; effective July 1, 2022. Available from https://www.acgme.org/Portals/0/ PFAssets/ProgramRequirements / 140 InternalMedicine 2022.pdf? ver=2021-02-19-152614-193. Accessed on March 15, 2021.

10. Colburn JL, Jasinski DR, Rastegar DA. Long-term opioid therapy, aberrant behaviors, and substance misuse: comparison of patients treated by resident and attending physicians in a general medical clinic. J Opioid Manag. 2012;8:153-160.

11. Roy $P$, Jackson $\mathrm{AH}$, Baxter $\mathrm{J}$, et al. Utilizing a faculty development program to promote safer opioid prescribing for chronic pain in internal medicine resident practices. Pain Med. 2019;20(4):707-716.

12. Alford DP, Carney BL, Brett B, et al. Improving residents' safe opioid prescribing for chronic pain using an objective structured clinical examination. Grad Med Educ. 2016;8(3):390-397.

13. Ruff AL, Alford DP, Butler R, et al. Training internal medicine residents to manage chronic pain and prescription opioid misuse. Subst Abus. 2017;38(2):200-204

14. Tesema L, Marshall J, Hathaway R, et al. Training in office-based opioid treatment with buprenorphine in US residency programs: A national survey of residency program directors. Subst Abus. 2018;39(4):434-440.

15. Wakeman SE, Baggett MV, Pham-Kanter G, et al. Internal medicine residents' training in substance use disorders: a survey of the quality of instruction and residents' self-perceived preparedness to diagnose and treat addiction. Subst Abus 2013;34(4):363-370.

16. Fletcher $\mathrm{KE}$, O'Connor $\mathrm{AB}$, Kisielewski $\mathrm{M}$, et al. Why do residency program directors consider resigning? A mixed-methods analysis of a national program director survey. Am J Med. 2020;133(6):761-767.

17. U.S. Census Bureau. Census Regions and Divisions of the United States. Available from https://www2.census.gov/geo/pdfs/maps-data/maps/ reference/us_regdiv.pdf. Accessed December 2019.

18. Accreditation Council for Graduate Medical Education. Accreditation Database System Online (Public). Available from https://apps.acgme. org/ads/Public/Programs/Search. Accessed June 1, 2018.

19. American Medical Association (AMA). Fellowship and Residency Electronic Interactive Database Access System (FREIDA) Online. Data provided via a licensing agreement between AMA and the Alliance for Academic Internal Medicine. Obtained September 1, 2019. Also available online at https://freida.ama-assn.org.

20. Yanni LM, McKinney-Ketchum JL, Harrington SB, et al. Preparation, confidence, and attitudes about chronic noncancer pain in graduate medical education. J Grad Med Educ. 2010;2:260-268.
21. Breuer B, Cruciani R, Portenoy R. Pain management by primary care physicians, pain physicians, chiropractors, and acupuncturists: a national survey. South Med J. 2010;108:738-747.

22. Yanni LM, Weaver MF, Johnson BA, et al. Management of chronic nonmalignant pain: a needs assessment in an internal medicine resident continuity clinic. J Opioid Manag. 2007;4:201-211.

23. Roth CS, Burgess DJ. Changing residents' beliefs and concerns about treating chronic noncancer pain with opioids: evaluation of a pilot workshop. Pain Med. 2008;9:890-902.

24. Smith CD. A curriculum to address family medicine residents' skills in treating patients with chronic pain. Int J Psychiatry Med. 2014;47:327-336.

25. Huhn AS, Dunn KE. Why aren't physicians prescribing more buprenorphine?. J Subst Abuse Treat 2017;78:1-7.

26. Andraka-Christou B, Capone MJ. A qualitative study comparing physician-reported barriers to treating addiction using buprenorphine and extended-release naltrexone in U.S. office-based practices. Int J Drug Policy. 2018;54:9-17.

27. Kirane H, Drits E, Ahn S, et al. Addressing the opioid crisis: An assessment of clinicians' training experience, practices, and attitudes within a large healthcare organization. J Opioid Manag 2019; 15(3): 193-204.

28. Egan JE, Casadonte P, Gartenmann T, et al. The Physician Clinical Support System-Buprenorphine (PCSS-B): a novel project to expand/ improve buprenorphine treatment. Gen Intern Med. 2010;25(9):936-941.

29. Boudreax DM, Lapham G, Johnson EA, et al. Documented opioid use disorder and its treatment in primary care across six U.S. health systems. J Subst Abuse Treat. 2020;112S:41-48.

30. Tong S, Sabo R, Aycock R, et al. Assessment of addiction medicine training in family medicine residency programs: A CERA study. Fam Med. 2017;49(7):537-543

31. Bowen JL, Irby DM. Assessing quality and costs of education in the ambulatory setting: a review of the literature. Acad Med. 2002;77(7):621-80.

32. Lin L, Peltzman T, McCarthy JF, et al. Changing trends in opioid overdose deaths and prescription opioid receipt among Veterans. Am J Prev Med. 2019;57(1): 106-10.

33. National Center for Complementary and Integrative Health Care. Partnering on Pain Research: NIH, DoD, and VA Announce Pain Management Collaboratory Funding Initiative January 4, 2017. Available from https://www.nccih.nih.gov/research/blog/partnering-on-pain-research-nih-dod-and-va-announce-pain-management-collaboratoryfunding-initiative. Accessed on February 28, 2021.

34. Wen H, Hockenberry JM, Borders TF, et al. Impact of Medicaid expansion on Medicaid-covered utilization of buprenorphine for opioid use disorder treatment. Med Care. 2017;55(4):336-341.

35. Vettese TE, Thati N, Roxas R. Effective chronic pain management and responsible opioid prescribing: aligning a resident workshop to a protocol for improved outcomes. MedEdPORTAL. 2018;14:10756. https://doi. org/10.15766/mep_2374-8265.10756

36. Matassa D, Perrella B, Feurdean M. A novel team-based learning approach for an internal medicine residency: medication-assisted treatments for substance use disorders. MedEdPORTAL. 2021;17:11085. https://doi.org/10.15766/mep_2374-8265.11085

37. Fujita AW, LaRosa A, Carter A. Treating withdrawal and pain in inpatients with opioid use disorder: a brief educational intervention for internal medicine residents. MedEdPORTAL. 2021;17:11123. https://doi.org/10. 15766/mep_2374-8265.11123

38. Boston University School of Medicine SCOPE (Safer/Competent Opioid Prescribing) of Pain. Available from https://www.scopeofpain.org. Accessed on July 24, 2021.

39. Haffajee RL, Lin LA, Bohnert ASB, Goldstick JE. Characteristics of US counties with high opioid overdose mortality and low capacity to deliver medications for opioid use disorder. JAMA Netw Open. 2019;2(6):e196373.

40. U.S. Department of Health and Human Services. HHS Releases New Buprenorphine Practice Guidelines, Expanding Access to Treatment for Opioid Use Disorder. April 27, 2021. Available from https://www.hhs. gov/about/news/2021/04/27/hhs-releases-new-buprenorphine-practice-guidelines-expanding-access-to-treatment-for-opioid-use-disorder. htmlAccessed on July 24, 2021.

Publisher's Note: Springer Nature remains neutral with regard to jurisdictional claims in published maps and institutional affiliations. 